\title{
Is nonperturbative inflatino production during preheating a real threat to cosmology?
}

\author{
${ }^{\dagger}$ Rouzbeh Allahverdi, ${ }^{\sharp}$ Mar Bastero-Gil and *Anupam Mazumdar \\ † Physik Department, TU Muenchen, James Frank Strasse, D-85748, Garching, Germany. \\ \# Scuola Normale Superiore, Piazza dei Cavalieri 7, 56126 Pisa, Italy \\ * ICTP, Strada Costiera 11, 34014 Trieste, Italy.
}

(November 3, 2018)

\begin{abstract}
We discuss toy models where supersymmetry is broken due to non-vanishing time-varying vacuum expectation value of the inflaton field during preheating. We discuss the production of inflatino the superpartner of inflaton due to vacuum fluctuations and then we argue that they do not survive until nucleosynthesis and decay along with the inflaton to produce a thermal bath after preheating. Thus the only relevant remnant is the helicity $\pm 3 / 2$ gravitinos which can genuinely cause problem to nucleosynthesis.
\end{abstract}

PACS numbers: 98.80.Cq TUM-HEP-396/00/, hep-ph/0012057

Inflation is perhaps one of the best paradigms of the early Universe which solves some of the nagging problems of the standard Big Bang cosmology [1]. One of the consequences of inflation is that it leaves the Universe extremely cold, virtually devoid of entropy. Thus, the Universe requires to be reheated to a temperature at least more than $\mathcal{O}(\mathrm{MeV})$ to keep the successes of the Big Bang nucleosynthesis. Perhaps, one can imagine that the Universe reheats via the decay of the scalar field whose potential has dominated the Universe during the inflationary regime. Inflation leaves the inflaton field extremely homogeneous except for the quantum fluctuations produced during inflation. The perturbations keep their imprint intact to match the observed anisotropy in the present Universe which is one part in $10^{5}$ [2]. Once inflation ends, the mass of the inflaton field dominates over the Hubble rate of expansion, and the homogeneous inflaton field oscillates coherently around the bottom of the potential. If we assume chaotic inflation with a massive inflaton field, $m$, and potential $V=m^{2} \phi^{2} / 2$, then, during the coherent oscillations the average pressure of the Universe within one Hubble time vanishes over many oscillations. As a result the decaying energy density of the Universe behaves as if it were in a matter dominated era with $\rho_{\phi}=\dot{\phi}^{2} / 2+m^{2} \phi^{2} / 2 \sim a^{-3}$, where $a$ is the scale factor of the expanding Universe. After couple of oscillations the energy density in the scalar field redshifts away in the same way as in the pressureless fluid but this does not lead to a radiation dominated Universe. To obtain a radiation dominated era, the inflaton field has to decay to other particles which will eventually lead to a thermalized plasma with a finite temperature, usually known as the reheat temperature of the Universe. The inflaton decays when the Hubble parameter $H \sim \Gamma_{\phi}$, where $\Gamma_{\phi}$ is the decay rate. The decay rate essentially depends on the kind of couplings the inflaton has to other particles 3 . However, in between the end of inflation and the beginning of the radiation era there can be an explosive production of particles purely due to non-thermal effects. This new wisdom has been realized in Refs. [4]. This is due to the fact that the oscillations in the inflaton field are extremely coherent and act as a Bose condensate fluid. So, in principle, one can study the quantum fluctuations of the inflaton quanta as well as bosonic and fermionic fields which are coupled to the inflaton field via Yukawa, gauge, or non-renormalizable couplings. Effectively, the problem turns out to be quantizing the bosonic and the fermionic fields in a time-varying inflaton background. This leads to an explosive production of particles which does not depend on the background temperature and it is purely an offshoot of a non-perturbative analysis. The production of bosons and fermions differs in its nature due to Pauli's exclusion principle, which prohibits excessive production of fermions compared to their bosonic counterparts [5]. In this regard, recently it has been realized that like fermions with spin $1 / 2$, other fermions with higher spin can also be created from the vacuum fluctuations in a time-varying scalar background. In Ref. [6], the authors have noticed that inspite of Planck mass suppressed couplings of spin $3 / 2$ particles to other fields, it is possible to excite them due to vacuum fluctuations. This has lead to many consequences which we briefly discuss in the next paragraph.

The spin 3/2 gravitino occurs in supersymmetric theories as a superpartner of the graviton. A massive spin $3 / 2$ has four helicity states $\pm 3 / 2$ and $\pm 1 / 2$. A massless gravitinos only possess $\pm 3 / 2$ helicity states. However, once supersymmetry is broken the gravitinos become massive, and they possess all four helicity states. In the early Universe supersymmetry can be broken due to non-zero vacuum contribution of the inflaton energy density. If the inflaton field is a scalar component of a chiral multiplet, then spontaneous supersymmetry breaking due to F-term leads to non-zero expectation value of the fermionic field [7].

$$
\left\langle 0\left|\delta_{\xi} \tilde{\phi}\right| 0\right\rangle=\left\langle-i \not \partial \phi \xi-e^{G / 2} G_{\phi} \xi\right\rangle \neq 0,
$$

where $\xi$ is the infinitesimal Grassmann-odd parameter, $\phi$ is a scalar field responsible for inflation, $\tilde{\phi}$ is the fermionic component of the inflaton in a single chiral field model, 
which we may call inflatino, $G$ is the Kähler function defined below, and $G_{\phi}$ is the derivative of the Kähler function with respect to the inflaton field. The Kähler function is defined by

$$
G=\frac{\phi_{i} \phi^{i *}}{\mathrm{M}^{2}}+\ln \left(\frac{|W|}{\mathrm{M}^{3}}\right),
$$

where we have assumed minimal Kähler function, $W$ is the superpotential and $\mathrm{M} \equiv \mathrm{M}_{\mathrm{p}} / \sqrt{8 \pi}$ is the reduced Planck mass. Out of the two terms present in the righthand side of Eq. (1), we notice that the first term gives a non-zero contribution during and after inflation, particularly during preheating. Therefore, the dynamical effects of the inflaton field breaks supersymmetry.

Soon after it has been realized that the helicity $\pm 3 / 2$ states of a massive gravitino can be produced nonperturbatively [6], it has been shown that the helicity $\pm 1 / 2$ states of a massive gravitino can also be produced from vacuum fluctuations [8]. However, they are more abundantly produced compared to that of the helicity $\pm 3 / 2$ states. This can be easily understood in a simple way. For the creation of particles from vacuum fluctuations, the adiabaticity condition has to be broken which is usually measured by a rate of change of a time-varying frequency of a given momentum mode. For fermions the frequency depends on an effective mass parameter. For example, for the helicity $\pm 3 / 2$, the mass parameter is essentially Planck mass suppressed. It has been noticed in Refs. [8], that the helicity $\pm 1 / 2$ states are massive due to the fact that they eat the mass of the fermionic component of the inflaton. This statement is true for a single chiral case and it has been pointed out that for helicity $\pm 1 / 2$ gravitinos, the adiabaticity condition is broken much more strongly compared to that of helicity $\pm 3 / 2$ gravitinos [9]. 12 .

All these results obtained were interesting because gravitino plays a key role in a standard Big Bang cosmology. If supersymmetry is required to solve the gauge hierarchy problem, then, in the gravity mediated supersymmetry breaking, the gravitino gets a mass around, $\mathcal{O}(\mathrm{TeV})$. Since their couplings to other particles are Planck mass suppressed, the life time of the gravitino at rest is quite long, $\tau_{3 / 2} \sim M_{\mathrm{p}}^{2} / m_{3 / 2}^{3} \sim$ $10^{5}\left(m_{3 / 2} / T e V\right)^{-3}$ sec, [13]. We know that successful nucleosynthesis depends on the baryon abundance: $Y_{\mathrm{B}}(T<$ $\mathrm{MeV}) \equiv n_{3 / 2} / n_{\gamma}=10^{-10} \sqrt{14}$. The gravitino decay products can easily change this ratio. Their decay products such as gauge bosons and its gaugino partners, or high energy photons, can generate a large entropy which will heat up the photons compared to $\tau$ and $\mu$ neutrinos. The abundance of neutrinos essentially determines the ${ }^{4} \mathrm{He}$ abundance. It was first pointed out in Ref. [15] that the gravitino mass must be larger than $\sim 10 \mathrm{TeV}$ in order to keep the successes of the Big Bang nucleosynthesis. On contrary, if the gravitinos were stable, and if their mass exceeded $1 \mathrm{KeV}$, they could easily overclose the Universe in absence of inflation [16]. However, after the end of in- flation the gravitinos can be produced from the thermal bath and this constraints the temperature of the thermal bath in order not to over produce them. At the time of nucleosynthesis the abundance is given in terms of the reheat temperature, $Y_{3 / 2}(T<\mathrm{MeV}) \sim 10^{-2}\left(T_{\mathrm{rh}} / M_{\mathrm{p}}\right)$ [17]. Thus, we see that there exists a strong constraint on the reheat temperature, $T_{\mathrm{rh}} \leq 10^{10} \mathrm{GeV}$, in order to maintain the baryon abundance one part in $10^{10}$ during nucleosynthesis. Since we know that non-perturbative creation of particles does not depend on temperature, it will be difficult to constrain a general parameter other than the model parameters. Hence, this leads to a natural suspicion that perhaps non-perturbative production of helicity $\pm 1 / 2$ gravitinos will cause a problem to nucleosynthesis.

The important point is that the inflaton has to completely decay to give rise to a thermal bath with a reheat temperature at least more than a $\mathcal{O}(\mathrm{MeV})$, and the fermionic component of the inflaton, known as inflatino, inevitably decays along with the inflaton. We know during the inflaton oscillations, the helicity $\pm 1 / 2$ states of the gravitino eat the mass of the inflatino, and they essentially behave as an inflatino when the amplitude of the inflaton oscillations has considerably dropped below $M_{P}$. As a result they must also decay along with the inflaton. As we shall see that this argument is quite robust and it should not depend if there were any other source of supersymmetry breaking other than the inflaton sector. During the preheating era of the Universe it is quite natural to think that the supersymmetry breaking due to the energy denisty stored in the inflaton oscillations is far the most dominant source.

We will begin with an introduction of a supersymmetric inflationary model with a single multiplet, and then we discuss decay rates of the inflaton and the inflatino in two models: namely with Planck mass suppressed coupling, and with Yukawa couplings to the visible sector. We then establish an equivalence between the helicity $\pm 1 / 2$ gravitino interactions to its supercurrent to that of the inflatino interactions in the supergravity Lagrangian when the amplitude of the inflaton oscillations are small compared to the Planck mass. In the last section we give a qualitative discussion upon the gravitino decay when more than one chiral fields are present.

\section{MODELS WITH A SINGLE MULTIPLET}

In the most part of this paper we shall focus on models where supersymmtery is broken by a single multiplet and also responsible for producing inflation in the early Universe. Nevertheless, to solve the low-energy (i.e. electroweak scale) supersymmetry breaking we may require some other sector, which can be a hidden sector, which we shall not take into account here. In our case the source of a time-varying supersymmetry breaking is the oscillations in the inflaton field $\phi$. During these oscillations the 
fermionic partner of the inflaton which we call here inflatino, whose mass is equal to the mass of the inflaton, is eaten by the helicity $\pm 1 / 2$ components of the gravitino to produce a massive gravitino. It has been suggested by many authors in Refs. 99,10, 12, that in a limit when $|\phi|<M_{\mathrm{p}}$, it is possible to use the inflatino mode equation to study the behavior of the helicity $\pm 1 / 2$ states of the gravitino. This can as well be understood from the point of view of an Equivalence Theorem (ET), which demands that when the energy scale $E \gg m_{3 / 2}(t)$, the wavefunction of the gravitino for the helicity $\pm 1 / 2$ components is approximately proportional to $p_{\mu} / m_{3 / 2}(t)$, where $p_{\mu}$ is the momentum of the gravitino and $m_{3 / 2}(t)$ is its timevarying effective mass. However, there is a word of caution regarding the validity of ET in our calculation during the oscillations of the inflaton. In principle, the timevarying mass of the gravitino can be larger than the momentum during the oscillations, or perhaps $m_{3 / 2}(t) \propto p_{\mu}$, and in both the cases ET cannot be trusted during the inflaton oscillations. However, studying the inflatino mode equation is not futile, because when the amplitude of the oscillations die down due to the expansion of the Universe, it is possible to identify the high momentum Fourier modes of the inflatino with those of the helicity $\pm 1 / 2$ gravitino. Therefore, only in those regions we can identify the inflatino to the helicity $\pm 1 / 2$ gravitinos (in a Fourier space), and we can therefore identify the Bogolyubov coefficients which are related to the number density of the produced helicity $\pm 1 / 2$ gravitinos. In this paper we are going to argue that ET can also be used to study the decay of the helicity $\pm 1 / 2$ gravitinos. However, this means that by using ET we shall be able to match the coupling strength of the helicity $\pm 1 / 2$ gravitinos to that of the inflatinos. This we shall discuss in the coming sections.

\section{A. Inflaton decaying via gravitational coupling}

As a first example we consider a new inflation model proposed in Ref. [18]. In this model the two distinct sectors are the inflaton sector and the visible sector. These sectors interact with each other only gravitationally, and can be considered separately in the superpotential. The construction of the inflaton sector demands supersymmetry is restored in the global minimum. While setting the cosmological constant to zero, the simplest form of a superpotential emerges 18

$$
\mathrm{I}=\frac{\Delta^{2}}{\mathrm{M}}(\Phi-\mathrm{M})^{2},
$$

where $\Delta$ determines the scale of inflation. Here we have denoted $\Phi$ as a superfield in the inflaton sector. The amplitude of the density perturbations produced during inflation by the inflaton, $\phi$, is fixed by the COBE scale, which constraints $\Delta / \mathrm{M} \approx 5 \times 10^{-3}$. With this choice of superpotential, inflation occurs for $\phi \ll \mathrm{M}$; the oscillations take place around the minimum of potential
$\phi_{0}=\mathrm{M}$, with a frequency $m_{\phi} \sim \Delta^{2} / \mathrm{M}$. The scalar potential derived from the above superpotential has a form

$$
\begin{array}{r}
V=e^{\sum_{j}\left(\left|\Phi_{j}\right| / \mathrm{M}\right)^{2}}\left(\sum_{k}\left|\frac{\partial W_{\mathrm{tot}}}{\partial \phi_{k}}+\frac{\phi_{k}^{*} W_{\mathrm{tot}}}{\mathrm{M}^{2}}\right|^{2}\right. \\
\left.-3 \frac{\left|W_{\mathrm{tot}}\right|^{2}}{\mathrm{M}^{2}}\right),
\end{array}
$$

where we have assumed minimal Kähler function and we consider the total superpotential to be

$$
W_{\mathrm{tot}}=\mathrm{I}+\mathrm{L}
$$

where L can be recognized as a visible sector which contains the light degrees of freedom. Before we begin our discussion on decaying inflaton, we mention some of the essential points related to this model. The dominant coupling of the inflaton to other light degrees of freedom can be read from the potential, Eq. (4). Just by expanding the interference term in Eq. (1), we notice that the inflaton field can decay only via trilinear coupling to the scalars. This certainly prevents creation of such scalar fields via parametric resonance. Hence, the decay of the inflaton is essentially perturbative in nature. Under the condition $m_{\phi}>H$, the decay rate of the inflaton does not depend upon the curvature of the universe. However, the inflaton field has a time varying amplitude, thus it is necessary to virialize the mean value of the field. Otherwise, we may expand the inflaton field around its minimum value $\mathrm{M}$, by assuming

$$
\phi^{\prime}=\phi-\mathrm{M}-\hat{\phi}(t)
$$

where $\hat{\phi}$ is assumed to have pure oscillatory part with an amplitude much less than one in units of reduced Planck mass. With the help of Eq. (6), it is easy to evaluate the interference terms coming from the first squared term in the bracket in Eq. (4). The leading order term in the expansion generates trilinear coupling to the matter sector from $\mathrm{L}$ with a gravitational strength $\sim \Delta^{2} / \mathrm{M}^{2}$, corresponding to a decay width $\Gamma_{\phi} \sim m_{\phi}\left(\Delta^{2} / \mathrm{M}^{2}\right)^{2}$. Since the mass of the inflaton is $m_{\phi} \sim \Delta^{2} / \mathrm{M}$, this gives a finite decay width of the inflaton [18]

$$
\Gamma_{\phi} \sim \frac{\Delta^{6}}{\mathrm{M}^{5}}
$$

If we assume that the inflaton energy is converted into radiation according to

$$
\rho_{\phi} \approx \frac{\pi^{2}}{30} g_{*} T_{\mathrm{r}}^{4}
$$

where $g_{*}$ is the relativistic degrees of freedom, then the reheat temperature of the Universe can be estimated by

$$
T_{\mathrm{r}} \sim\left(\frac{30}{\pi^{2} g_{*}}\right)^{1 / 4}\left(\Gamma_{\phi} \mathrm{M}\right)^{1 / 2} \approx 10^{-1} \frac{\Delta^{3}}{\mathrm{M}^{2}}
$$


For $\Delta / \mathrm{M} \sim 5 \times 10^{-3}$, the reheat temperature is around $T_{\mathrm{r}} \sim 10^{8} \mathrm{GeV}$.

With this introduction we may now turn our attention to the decay of the helicity $\pm 1 / 2$ gravitinos which are created during the oscillations of the inflaton field from the vacuum fluctuations. We remind that gravitino production is completely non-thermal, and we cannot associate their number density to any particular thermal bath. We also notice that the mass of the gravitino need not necessarily be that of a gravitino mass around $\mathcal{O}(\mathrm{TeV})$. Especially, if the inflaton sector has a supersymmetric preserving minimum with a zero cosmological constant, then the mass of the gravitino vanishes after the end of reheating, provided there is no other source of supersymmetry breaking in any other sector. However, the situation can be little bit different if there exists other sources of supersymmetry breaking. This we shall briefly discuss in the next section. In the present section we shall concentrate upon the former case where we argue that whatever helicity $\pm 1 / 2$ gravitinos are created during preheating shall have to decay along with the inflaton to have a successful Big Bang nucleosynthesis 7 .

The equation of motion for the helicity $\pm 1 / 2$ gravitino in a cosmological background has been derived in the literature by using alternative approaches [ 8 12]. The important realization is that when the amplitude of the oscillations is much smaller than the reduced Planck mass, the equation of motion for the helicity $\pm 1 / 2$ gravitino is effectively that of the goldstino in a global supersymmetric limit. For a single chiral case the goldstino is equivalent to the inflatino up to a phase. Here we simply quote some of the useful formulae which have already been established in Refs. [8,9, 12]. The evolution of the inflatino, which we define here as $\tilde{\phi}$, is given by [9]

$$
i \gamma^{0} \dot{\tilde{\phi}}-\hat{k} \tilde{\phi}-m_{\text {eff }} \tilde{\phi}=0
$$

where $\hat{k}=\gamma^{i} k_{i}$, and $k_{i}$ are components of the physical momentum, while $\gamma^{i}$ are the gamma matrices. The

\footnotetext{
* Here we would like to point out that eventhough we are considering inflaton to be the only source of supersymmetry breaking which might seems unrealistic at first point but the analysis is much simpler in this case and our arguments hold true even if there is another source of supersymmetry breaking. It is possible to consider a Polnyi sector which breaks supersymmtry in the hidden sector of the theory but as we shall see in the next section that its mass contribution to the mass of the goldstino is very small and of the order of $\mathrm{TeV}$. As a result the goldstino mass is essentially dominated by the inflatino mass. In such a circumstance our present analysis of single chiral field is quite general and as long as supersymmetry breaking due to the inflaton sector dominates over any other sector which is quite normal to think, we can apply our results faily well. All that we require is that the helicity $\pm 1 / 2$ gravitino mass is essentially being contributed by the inflatino mass.
}

validity of the above equation holds only in a global supersymmetric limit.

When the amplitude of the inflaton oscillations $|\phi| \ll$ $\mathrm{M}$, the effective mass of the helicity $\pm 1 / 2$ gravitinos, for a single chiral field and after phase rotation of the helicity $\pm 1 / 2$ gravitino field, is simply the mass of the fermionic component of the inflaton field, which yields [9]

$$
m_{\mathrm{eff}}=\frac{\partial^{2} \mathrm{I}}{\partial \phi^{2}}
$$

where $\mathrm{I}$ is the inflaton superpotential. For a simple superpotential Eq. (3), the effective mass for the helicity $\pm 1 / 2$ gravitino turns out to be equal to

$$
m_{ \pm 1 / 2} \simeq m_{\mathrm{eff}} \sim \frac{\Delta^{2}}{\mathrm{M}},
$$

which is the same as the mass of the inflaton [18]. On the other hand, for the same superpotential the other helicity $\pm 3 / 2$ gravitinos have mass given by [7]

$$
m_{ \pm 3 / 2} \equiv e^{\phi^{2} / 2 \mathrm{M}^{2}} \frac{|\mathrm{I}|}{\mathrm{M}^{2}} \sim \frac{\Delta^{2}}{\mathrm{M}}\left(\frac{\phi(t)}{\mathrm{M}}\right)^{2}
$$

where we have assumed that the visible sector $\mathrm{L}$ does not contribute to the gravitino mass. This is quite apparent from the above expressions Eqs. (12, 13), that the mass of the helicity $\pm 3 / 2$ gravitinos is not only suppressed by the reduced Planck mass, but it also contains time varying amplitude of the oscillations; $\sim \phi(t)$, which becomes vanishingly small near the bottom of the potential. This is quite obvious because mass of the helicity $\pm 3 / 2$ gravitinos is essentially generated by the dynamics of the inflaton field, and it must vanish when supersymmetry is restored at the global minimum of the potential. Before we begin our discussion on the decay of gravitinos, we compare different mass scales with the Hubble expansion. For the superpotential Eq. (3), the Hubble parameter it is given by $H \sim\left(\Delta^{2} / \mathrm{M}\right)(\phi(t) / \mathrm{M})$. This leads to a simple inequality in various mass scales which we must bear in mind

$$
m_{\phi} \approx m_{ \pm 1 / 2}>H>m_{ \pm 3 / 2} .
$$

\section{B. Inflatino interactions}

In this subsection we analyze the decay rate of the inflatino. We consider a following interaction which can be found in the matter Lagrangian [7]

$$
|\operatorname{det} e|^{-1} \mathcal{L}=-\frac{1}{2} e^{G / 2} G^{i} G^{j} \bar{\chi}_{i} \chi_{j \mathrm{~L}}+\text { h.c. },
$$

where $G^{i}$ is the derivative of the Kähler potential with respect to left and right chiral components. We can fix the index; $i=\phi$, corresponding to the inflaton sector. 
This leaves the other index $j$ to run on the chiral components of the visible sector L. Now according to our previous discussion on the inflaton decay, here again, we may assume that the inflatino is decaying into particles and sparticles of the sector $\mathrm{L}$ with an interaction of the form $\tilde{\phi} \chi_{j} \phi_{j}$, where $\tilde{\phi}$ is the inflatino, $\chi_{j}$ is the fermionic component, and $\phi_{j}$ is the bosonic fields belonging to the sector L. The decay is essentially mediated via gravitational coupling strength $\sim \Delta^{2} / \mathrm{M}^{2}$. This corresponds to a decay width of the inflatino with mass $\sim \Delta^{2} / \mathrm{M}$, which yields

$$
\Gamma_{\tilde{\phi}} \approx \frac{\Delta^{6}}{\mathrm{M}^{5}} .
$$

This decay rate is the same as the decay rate of the inflaton. The result is not surprising because the inflatino mass is similar to the inflaton mass, and both interact gravitationally to the visible sector particles. Now, if we argue that the helicity $\pm 1 / 2$ states of the gravitino essentially behave as an inflatino in a global supersymmetric limit, which corresponds to demanding that the amplitude of the inflaton oscillations $|\phi| \ll \mathrm{M}$, then and only then, we may argue that the helicity $\pm 1 / 2$ gravitinos decay along with the inflaton into visible sector particles. This is an important and generic conclusion which bears more attention. Our statement is only true provided we believe in the equivalence between inflatino and the helicity $\pm 1 / 2$ gravitinos at late stages of the inflaton oscillations, i.e. when Eq. (14) is satisfied.

Intuitively, our result makes sense, because if supersymmetry is restored at the bottom of the potential in the absolute minimum, then, only the $\pm 3 / 2$ components of the gravitino should survive, and not the $\pm 1 / 2$ components of the gravitino. Thus, the helicity $\pm 1 / 2$ states must decay along with the inflaton decay. This situation could have been different if there were a hidden sector, which was responsible for supersymmetry breaking at an intermediate scale, which would then communicated to the visible sector at low scale. This we shall discuss in the last section. So far we have studied only the inflatino interactions. However, to be more concrete we must study the gravitino interactions which we shall discuss in the next subsection.

\section{Interactions of the gravitino}

The gravitino interaction terms appear from the couplings between the gravitino field and the supercurrent

$$
\begin{array}{r}
\mathcal{L}_{\psi J}=\frac{1}{\sqrt{2} \mathrm{M}} \bar{\Psi}_{\mu} \not D \phi^{* j} \gamma^{\mu} \chi_{j \mathrm{~L}}+\frac{i}{\sqrt{2} \mathrm{M}} e^{G / 2} G^{i} \bar{\Psi}_{\mu} \gamma^{\mu} \chi_{i \mathrm{~L}} \\
+ \text { h.c. }
\end{array}
$$

where $\mu$ stands for the space-time index, $\chi_{i}$ is a fermionic field and $\phi^{i}$ is a bosonic field. Here the subscripts $i, j$ correspond to the visible sector $\mathrm{L}$, which contains the light degrees of freedom. We have neglected the vector multiplets in the above equation and assumed $\phi$ to be homogeneous. The total derivative $D_{\mu}$ is defined by

$$
D_{\mu}=\partial_{\mu}+\frac{1}{2} \omega_{\mu a b} \sigma^{a b}
$$

where $\omega_{\mu a b}$ is the spin connection.

It is to be mentioned that apart from the derivative coupling of the chiral field to the gravitino, we have an extra interaction term which is not usually considered otherwise. In fact the interaction terms proportional to $\gamma^{\mu} \Psi_{\mu}$ are usually not necessary in a static limit of the background field (i.e. inflaton field ), because $\gamma^{\mu} \Psi_{\mu}=0$ acts as a constraint for a gravitino field in a static background. However, this need not be true in a non-static background. It has been shown that in an expanding Universe, and in a time-varying inflaton background, $\pm 1 / 2$ helicity states follow $\gamma_{\mu} \Psi^{\mu} \neq 0$ [8]. Although, the same constraint continues to hold good for the helicity $\pm 3 / 2$ components of the gravitino in the same background along with the Dirac equation [6]. Thus, both the terms in Eq. (17) should be taken into account to study the efficient decay of the gravitino. In this subsection we will study the decay by assuming the validity of the equivalence between the helicity $\pm 1 / 2$ states of the gravitino and that of the inflatino at late stages of oscillations, i.e. when Eq. (14) is satisfied.

After several oscillations of the inflaton field $|\phi| \ll \mathrm{M}$, or, equivalently $H \ll m$. Note that under this condition, the kinematics of the inflaton, such as decay rate does not depend on the curvature of the Universe. As a result of this the decay rate of the inflaton coicides with that of the flat space-time limit. All the fields whose effective mass is larger than the Hubble parameter during the oscillations of the inflaton would actually not feel any effect of curvature of the Universe. Since, the effective mass of the helicity $\pm 1 / 2$ gravitino is similar to the mass of the inflaton, and, it is much larger than the Hubble parameter, suggests that we can study their evolution by neglecting the curvature of the Universe. Therefore, we replace $\pm 1 / 2$ helicity of the gravitino by an ansatz

$$
\Psi_{\mu} \sim \sqrt{\frac{2}{3}} \frac{\mathrm{M}}{\rho_{\phi}^{1 / 2}} \partial_{\mu} \eta,
$$

where $\eta$ represents the spin $1 / 2$ fermionic field, which we shall interpret as a goldstino instead of inflatino. At this moment this prescription seems to be unwarranted, but, we shall see that this choice of derivative wavefunction leads to the interactions of the helicity $\pm 1 / 2$ gravitino to that of the inflatino. This prescription has also been used in Ref. [9]. The goldstino is however related to the inflatino by a phase factor, and, it is expressed in Eq. (27). The above expression is exactly the wavefunction of the helicity $\pm 1 / 2$ gravitino in terms of goldstino in the limit when the energy scale of the gravitino is larger than its effective mass. This expression has been previously used 
in Refs. [19 21, where the authors have been studying the scattering processes of the helicity $\pm 1 / 2$ gravitino in a limiting case when the energy scale of the gravitino is larger than its mass in a flat space-time. Here, again we have a similar situation where the helicity $\pm 1 / 2$ gravitino does not feel the Hubble expansion, however, the only difference is that now supersymmetry is broken due to the oscillating scalar field rather than the static vacuum contribution. This obviously makes the difference. Notice, that instead of the mass of the gravitino $m_{3 / 2} \sim 1 \mathrm{TeV}$, now we have $\rho_{\phi}^{1 / 2} / M$, this is precisly because of the reason we have mentioned above, here supersymmetry is effectively broken due to the presence of the finite energy contribution of the inflaton field. The oscillations of the inflaton field also ensures that one has to include the interactions which are also proportional to $\gamma^{\mu} \Psi_{\mu}$. Another fact that the equation of motion of helicity $\pm 1 / 2$ gravitino is the same as that of the goldstino for $H \ll m$, as indicated in Refs. [8,9], further supports our approximation. We reiterate that we shall always work in a limit $\phi / \mathrm{M} \ll 1$.

Substituting Eq. (19), in Eq. (17), we derive an effective Lagrangian. Which yields

$$
\begin{gathered}
\mathcal{L}_{\text {eff }}=\frac{1}{\sqrt{3} \rho_{\phi}^{1 / 2}} \partial_{\nu} \varphi^{*} \partial_{\mu} \bar{\eta} \gamma^{\nu} \gamma^{\mu}\left(\frac{1+\gamma_{5}}{2}\right) \chi \\
+\frac{i}{\sqrt{3} \rho_{\phi}^{1 / 2}} m_{\chi} \varphi^{*} \partial_{\mu} \bar{\eta} \gamma^{\mu}\left(\frac{1+\gamma_{5}}{2}\right) \chi+\text { h.c. }
\end{gathered}
$$

where $\varphi$ denotes the bosonic field in a visible sector $\mathrm{L}$, the spinor component is defined by $\chi$; its mass can be written as

$$
m_{\chi} \approx e^{\phi^{2} / 2 \mathrm{M}^{2}} \frac{\partial^{2} \mathrm{~L}}{\partial \varphi^{2}} .
$$

We will use $m_{\chi}$ quite often to compactify our notations. In fact the mass of the fermion need not be a constant and can have field dependency. We can simplify Eq. (20) if we follow the below mentioned identities for the Majorana spinors

$$
\begin{aligned}
\bar{\eta} \gamma^{\mu} \chi & =-\bar{\chi} \gamma^{\mu} \eta \\
\bar{\eta} \gamma^{\mu} \gamma^{5} \chi & =\bar{\chi} \gamma^{\mu} \gamma^{5} \eta \\
\bar{\eta} \gamma_{5} \chi & =\bar{\chi} \gamma_{5} \eta
\end{aligned}
$$

With the help of Eq. (22), we can derive an effective Lagrangian after some algebraic manipulation, which reads

$$
\begin{aligned}
\mathcal{L}_{\mathrm{eff}}= & \frac{1}{\sqrt{3} \rho_{\phi}^{1 / 2}}\left[\left(m_{\varphi \mathrm{R}}^{2}-m_{\chi}^{2}\right) \varphi_{\mathrm{R}} \bar{\eta} \chi-i \frac{\partial m_{\chi}}{\partial t} \varphi_{\mathrm{R}} \bar{\eta} \gamma^{0} \chi\right] \\
& -\frac{i}{\sqrt{3} \rho_{\phi}^{1 / 2}}\left[\left(m_{\varphi \mathrm{I}}^{2}-m_{\chi}^{2}\right) \varphi_{\mathrm{I}} \bar{\eta} \gamma_{5} \chi+i \frac{\partial m_{\chi}}{\partial t} \varphi_{\mathrm{I}} \bar{\eta} \gamma^{0} \gamma_{5} \chi\right] \\
+ & \text { h.c. }+ \text { total derivative },
\end{aligned}
$$

where $m_{\varphi \mathrm{R}}$ denotes the real part of the light bosonic field $\varphi_{\mathrm{R}}$ residing in the sector $\mathrm{L}$. While deriving the above expression we have neglected the time derivative of the energy density. Eq. (23) can be further simplified if we assume that the mass splitting between $\varphi$ and $\chi$ is due to supersymmetry breaking by the inflaton oscillations. To simplify the situation we will be assuming that the visible sector must contain the quadratic terms in the superpotential. This can be written as

$$
\mathrm{L}=\frac{1}{2} \frac{\partial^{2} \mathrm{~L}}{\partial \Lambda^{2}} \Lambda^{2}+\ldots,
$$

where $\Lambda(\varphi, \chi)$ denotes the superfield, and ... terms can contribute due to other possibilities in the superpotential, which we shall not take into account here. Now we will explicitly show that if the inflaton sector and the visible sector interacts gravitationally, then it is possible to derive an effective inflatino Lagrangian which will have a similar coupling to Eq. (15). To get the desired result we first need to know the mass splitting between the fields of the visible sector. To get the mass splitting we expand Eq. (4), with the help of Eqs. (5) and (24), while considering only the dominant terms in the potential which is due to the interference terms. We also take help of Eq. (21) to obtain

$$
m_{\varphi \mathrm{R}}^{2}-m_{\chi}^{2} \approx e^{\phi^{2} / 2 \mathrm{M}^{2}}\left\langle\frac{\phi}{\mathrm{M}}\right\rangle\left\langle\frac{1}{\mathrm{M}} \frac{\partial \mathrm{I}}{\partial \phi}\right\rangle m_{\chi},
$$

and, similar expression holds for $m_{\varphi \mathrm{I}}^{2}-m_{\chi}^{2}$, except for the negative sign on the right hand side. Similarly, one may also obtain

$$
\frac{\partial m_{\chi}}{\partial t} \approx\left\langle\frac{\dot{\phi}}{\mathrm{M}}\right\rangle\left\langle\frac{\phi}{\mathrm{M}}\right\rangle m_{\chi} .
$$

We also notice that the goldstino can be expressed as in Refs. 99.11, 12

$$
\eta=\frac{1}{\rho_{\phi}^{1 / 2}}\left(i \gamma^{0} \frac{\partial \phi}{\partial t}-e^{\phi^{2} / 2 \mathrm{M}^{2}} \frac{\partial \mathrm{I}}{\partial \phi}\right) \tilde{\phi}
$$

where inflatino is represented by $\tilde{\phi}$. Here we have explicitly used the fact that the dynamics of the inflaton is breaking supersymmetry. In Eq. (27), we have only retained the leading order terms and neglected $\mathcal{O}\left(1 / \mathrm{M}^{2}\right)$ terms. Now, with the help of Eqs. (25, 26,27), we simplify Eq. (23)

$$
\mathcal{L}_{\text {eff }}=m_{\chi} \frac{\phi}{\mathrm{M}^{2}} \varphi_{R} \overline{\tilde{\phi}} \chi+i m_{\chi} \frac{\phi}{\mathrm{M}^{2}} \varphi_{I} \overline{\tilde{\phi}} \gamma_{5} \chi+\text { h.c. }
$$

where we have used the identity $\gamma^{0 \dagger}=\gamma^{0}$, and the fact that the following relation holds

$$
\begin{array}{r}
\left(-i \gamma^{0} \frac{d \phi}{d t}-e^{\phi^{2} / 2 \mathrm{M}^{2}} \frac{\partial \mathrm{I}}{\partial \phi}\right)\left(e^{\phi^{2} / 2 \mathrm{M}^{2}} \frac{\partial \mathrm{I}}{\partial \phi}-i \gamma^{0} \frac{d \phi}{d t}\right) \\
=-\rho_{\phi} .
\end{array}
$$

Now it is interesting to note that Eq. 28, upto a leading order actually leads to a familiar form 


$$
\mathcal{L}_{\text {eff }} \approx e^{G / 2} \frac{\partial G}{\partial \phi} \frac{\partial G}{\partial \varphi} \overline{\tilde{\phi}} \chi_{\mathrm{L}}+\text { h.c. }
$$

which is the inflatino coupling in Eq. (15). While deriving the above expression we have assumed Eq. (21). This clearly indicates that at late time of the inflaton oscillations when we recognize the helicity $\pm 1 / 2$ component of the gravitino as a goldstino, we essentially get similar coupling to the visible sector as that of the inflatino. This is the most important equivalence which establishes the fact that, since, for any successful inflationary model inflaton has to decay, and, so does the inflatino, the helicity $\pm 1 / 2$ component of the gravitino must also decay if the inflaton oscillations is the only viable source of supersymmetry breaking at that time. Our result is strictly correct for a single chiral field responsible for supersymmetry breaking. A further generalization to multi-chiral field supersymmetry breaking is more involved and we leave this for our future investigation.

Now, we move onto a toy model where the inflaton sector and the visible sectors are coupled via Yukawa couplings. We will establish similar result as we have already obtained in this section.

\section{Model with a Yukawa coupling to the Inflaton}

As a second example we consider a model with a following superpotential

$$
W=\frac{1}{2} m \Phi^{2}+\frac{1}{2} h \Phi \Sigma^{2},
$$

where $\Phi$ contains the inflaton field, which is responsible for the slow-roll inflation. However, now the inflaton field has an explicit Yukawa coupling to the matter sector given by the second term in Eq. (31). Such a coupling will enable inflaton to decay much more efficiently. Such a superpotential leads to interaction terms $h m \phi \sigma \sigma, h \phi \tilde{\sigma} \tilde{\sigma}$, $h \tilde{\phi} \tilde{\sigma} \sigma$, where $\phi$ is the inflaton field, $\tilde{\phi}$ is the inflatino, $\sigma$ is a light bosonic field, and its fermionic partner has been denoted by $\tilde{\sigma}$. The estimated rate of the inflaton decaying to fermionic component $\tilde{\sigma} \tilde{\sigma}$ is given by $\Gamma_{\phi} \sim\left(h^{2} / 8 \pi\right) m$.

In general the Yukawa coupling between $\Phi$ and $\Sigma$ multiplets can also result in the oscillations along the $\sigma$ field. If the $\sigma$ field eventually decays into other products much before the oscillations in $\sigma$ commences, then, it can still be a viable model to imagine that supersymmetry is broken by the inflaton field only. But in general, this may lead to a more complicated situation where supersymmtery is broken by several multiplets. However, it is possible to prevent this provided we require that the $\phi$-induced mass to the $\sigma$ field is much smaller than the Hubble expansion, i.e. $h \phi<H$, which implies $h<m / \mathrm{M}$. We note that this will also insure that $\sigma$ and $\tilde{\sigma}$ are not produced via parametric resonance. A viable choice of parameters which can lead to an inflationary paradigm for the $\phi$ field in a quadratic potential are; $m=10^{13}$ $\mathrm{GeV}$, and, a small Yukawa coupling $h=10^{-7}$, which ensures that at late stages of the inflaton oscillations, $\phi / \mathrm{M} \leq 10^{-14}$, the inflaton is decaying perturbatively. Following our previous discussion, again, we argue here that since the inflatino mass is same as that of the mass of the inflaton, and, if the helicity $\pm 1 / 2$ components of the gravitino is recognized as inflatino at late stages of the inflaton oscillations, then they must decay to $\sigma$, or $\tilde{\sigma}$ via a Yukawa coupling.

So far, we have been looking upon direct inflatino coupling to $\sigma$ and $\tilde{\sigma}$. However, we may now repeat the same analysis as we have shown earlier that indeed the helicity $\pm 1 / 2$ components of the gravitino has a similar coupling as that of the inflatino by using the equivalence theorem. The generalization is quite simple and we recognize that

$$
\frac{\partial^{2} W}{\partial \Sigma^{2}}=h \Phi
$$

where $\Sigma$ and $\Phi$ are the superfields denoted in Eq. (31). The mass of the fermion in this model is given by $m_{\tilde{\sigma}} \approx$ $e^{\phi^{2} / 2 \mathrm{M}^{2}} h\langle\phi\rangle$. Following our earlier argument we can find out the leading order contribution to the mass splitting, which yields

$$
m_{\sigma \mathrm{R}}^{2}-m_{\tilde{\sigma}}^{2} \approx h\left\langle\frac{\partial \mathrm{I}}{\partial \phi}\right\rangle .
$$

where I $\equiv(1 / 2) m \Phi^{2}$. Similarly, one can also derive an expression for $m_{\sigma \mathrm{I}}^{2}-m_{\tilde{\sigma}}^{2}$, which differs from the above by a negative sign. While deriving Eq. (33), we have neglected the Planck mass suppressed contributions which would anyhow be insignificant at late times. The analogue of Eq. (26) can be expressed as

$$
\frac{\partial m_{\tilde{\sigma}}}{\partial t} \approx h\left\langle\frac{\partial \phi}{\partial t}\right\rangle,
$$

and, one can also derive an effective Lagrangian with the help of Eqs. (23), (29), (33) and (34). Which yields

$$
\mathcal{L}_{\text {eff }} \sim h\left(\sigma_{\mathrm{R}} \overline{\tilde{\phi}} \tilde{\sigma}+i \sigma_{\mathrm{I}} \overline{\tilde{\phi}} \gamma_{5} \tilde{\sigma}\right)+\text { h.c. }
$$

where inflatino is denoted by $\tilde{\phi}$. After some calculation it can be shown that Eq. (35), actually leads to an expression

$$
\mathcal{L}_{\text {eff }} \sim h \sigma^{*} \overline{\tilde{\phi}} \tilde{\sigma}_{\mathrm{R}}+\text { h.c. } .
$$

This reinsures our earlier claim that the equivalence between the helicity $\pm 1 / 2$ gravitino and the goldstino is viable at late times of the inflaton oscillations. This equivalence is not only important for studying the production of the helicity $\pm 1 / 2$ components of the gravitino, but also describing the decay of the helicity $\pm 1 / 2$ gravitinos.

So far, we have not spoken any word on the other helicity states of the gravitino, namely $\pm 3 / 2$. The reason is it is extremely difficult to study their decay, precisely because the mass of the helicity $\pm 3 / 2$ is solely due to the dynamics of the inflaton field [6]. Their effective mass is 
Planck mass suppressed, and also depends on the amplitude of the oscillations of the inflaton field. This leads to an obvious result that if there is no other source of supersymmetry breaking other than the inflaton oscillations, then, the effective mass for the helicity $\pm 3 / 2$ component should vanish at the end of reheating. It is difficult to make a precise calculation for the decay of the helicity $\pm 3 / 2$ gravitinos. However, we believe that their survival does not depend on the inflaton decay as they have no goldstino nature. Next, we discuss qualitatively what would happen if the hidden sector supersymmetry breaking is also taken into account.

\section{MODELS WITH SEVERAL MULTIPLETS}

Once we invoke more than one sectors, and treat them at equal level, the problem of gravitino production becomes more complicated. This problem has been addressed in Refs. 9, 11, 12] to some extent, and yet lot to be understood in this direction. In this case it has been realized that the goldstino is a linear combination of all the fermions, and as a result, even if we use the goldstinogravitino equivalence we cannot in general guarantee that a major contribution to the goldstino mass is coming from the fermionic component of the inflaton. There are some interesting cases where the multi field case can be expressed as a single field, such as supersymmetric hybrid inflation model where effectively the two fields behave as if there were a single degree of freedom [11]. In such a model it is possible to extract the goldstino mass, which is again of the order of the inflaton mass. One can then discuss the decay rate of the inflatino in this model [11, and the inflatino decay rate to the light degrees of freedom would exactly be the same as that of the inflaton. Interesting question would be to address a problem where there exists a hidden sector which is responsible for supersymmetry breaking in that sector, and also responsible for mediating supersymmetry breaking gravitationally to the observable sector. In such a case the gravitino will have an effective mass $\sim \mathcal{O}(\mathrm{TeV})$ at a low energy scale. So, keeping this in mind we may consider a simple toy model with a following superpotential

$$
W=\frac{1}{2} m_{1} \Phi^{2}+m_{2}^{2}[Z+(2-\sqrt{3}) \mathrm{M}],
$$

where $\Phi$ and $Z$ are inflaton and Polonyi multiplets respectively. We assume that $\phi$ field is responsible for inflation, so we set $m_{1}=10^{13} \mathrm{GeV}$ to produce adequate density perturbation, while setting $m_{2}=10^{11} \mathrm{GeV}$ for giving an effective mass to the gravitino around $\mathcal{O}(\mathrm{TeV})$. An interesting discussion regarding this model has been sketched in Ref. [12].

A serious difficulty which immediately arises is that one derives a set of coupled equations for the helicity $\pm 1 / 2$ gravitino and other fermionic degrees of freedom [9,12]. It has been shown in Ref. [12], that in a global supersymmetric limit, this set of equations is reduced to a coupled set of equations for the goldstino and the transverse combination of the fermions. This suggests that there exists a mixing between the goldstino and the transverse combination of the fermions. As a result one cannot describe the goldstino in a mass eigenstate, and thus, it is also difficult to estimate the evolution of their number densities. There are many technical difficulties because there are essentially two time scales in the problem. The first one is related to the fact that the effective mass scale of the bosons oscillating and exciting the fermionic modes, and, the other one is related to the mixing between the goldstino and the transverse combination of the fermions (for details, we refer the readers Refs. [9, 12]). In general one can derive a relationship between the two time scales, but this is a non-trivial task and we do not have enough tools to address this problem.

For the above superpotential Eq. (37), the inflaton and the Polonyi sectors have only gravitational interactions. The fermionic components $\tilde{\phi}$ and $\tilde{z}$ have masses $m_{1}$ and zero respectively in the global supersymmetric limit. The goldstino in this model is a linear combination of the fermionic components from both the sectors. As long as the energy density is dominated by the inflaton field, the helicity $\pm 1 / 2$ gravitinos essentially behave as an inflatino, because the mass contribution to the goldstino from the Polnyi sector is much smaller $\sim \mathcal{O}(\mathrm{TeV})$. This particular case is quite interesting and we can analyse the decay of the gravitino by assuming that the gravitinos are created from the vacuum fluctuations due to the inflaton oscillations, whose energy density is dominating the Universe. The helicity $\pm 1 / 2$ gravitinos produced during preheating will essentially decay because they are essentially the inflatino components and so their couplings are determined in the same fashion as that of the inflaton.

However, the energy density in the inflaton sector is decreasing in time, and, when the Hubble expansion $H<\mathcal{O}(\mathrm{TeV})$, the $\tilde{z}$ component dominates the goldstino. Usually, the mixing between the inflatino and $\tilde{z}$ is minimal and Planck mass suppressed, so, the fermions which are produced during preheating will decay again in the form of inflatino and cause no trouble for nucleosynthsis, yet there is a finite probability to mix the fermionic states and conversion of inflatino to the fermionic partner of the Polonyi field. Though, we shall not discuss this possibility in this paper. One can also imagine that the oscillations in the Polonyi sector are also induced at $H \approx \mathcal{O}(\mathrm{TeV})$. Once, $z$ field starts oscillating, one might expect supersymmetry is broken by the oscillations in $z$ direction also, and, as a result gravitinos can as well be excited. One may also suspect that the late production of the helicity $\pm 1 / 2$ gravitinos will dominate and the problem of gravitino decay still persists. The suspicion is not fully correct because the number density of the helicities $\pm 1 / 2$ and $\pm 3 / 2$ are more or less equal now. This is because superpotential contribution to the mass of the fermionic component of the Polonyi field is very small $\sim \mathcal{O}(\mathrm{TeV})$ and the only time-varying scale is due 
to time-varying mass of the gravitino $\sim e^{z z^{*} / 2 \mathrm{M}^{2}}|W| / \mathrm{M}^{2}$. The presence of the Planck mass suppression prohibits explosive production of the gravitinos at late times, so especially in the model we have considered, the late time production of helicity $\pm 1 / 2$ can not be very abundant. But, now the problem could be much more severe, because these gravitinos with both the helicities are produced much later, and their effective masses are also very small roughly of the order of TeV. This leads to extremely slow decay rate of these gravitinos which may cause a problem to the Big Bang nucleosynthesis. This picture is similar to late production of gravitinos discussed in Refs. [22]. Furthermore, the oscillating Polonyi field leads to an even more serious problem, i.e. the moduli problem, of which there is no satisfactory way out.

Finally, we mention and also pointed out in Ref. [12], that if the fermionic components mix freely, the inflatinos can be converted to $\tilde{z}$ (which is the field eventually eaten by the gravitino). This presumably occurs around the time when contributions to supersymmtery breaking from the inflation sector and the Polonyi sector become comparable. This problem is analogue to the neutrino flavor conversion and the relevant question is to ask the conversion probability. As mentioned, we beileve that an effcicient conversion will not take place for the Polonyi model. An efficient conversion nevertheless results in a large abundance (i.e. comparable to the abundances which are produced during preheating) of $\tilde{z}$ fermion, on top of what is produced due to oscillations of the Polonyi field 7 . We notice that if the inflatino decays before $H \approx \mathcal{O}(\mathrm{TeV})$, then the abundance of the inflatinos prior to conversion will decrease leading to a smaller abundance for $\tilde{z}$ (and consequently helicity $\pm 1 / 2$ gravitinos) even after an efficient conversion. The quantitative analysis is beyond the scope of this paper and we leave that for future investigation.

\section{CONCLUSION}

Our main result of this paper is to show that the inflatino coupling to the matter field is similar to that of the helicity $\pm 1 / 2$ gravitinos. This merely confirms that the gravitino interaction with the supercurrent actually leads to the same interactions as that of the inflatinos when the amplitude of the inflaton oscillations is small $|\phi| \ll \mathrm{M}$, under the assumption that the helicity $\pm 1 / 2$ component of the gravitino behaves as a goldstino for a momentum larger than the gravitino mass in a time-varying background. Then we have argued that the production of helicity $\pm 1 / 2$ states of the gravitino, especially for models where supersymmetry breaking scale is dominated by

\footnotetext{
${ }^{\dagger}$ This is the abundance of $\tilde{z}$ fermion which will eventually determine the abundance of helicity $\pm 1 / 2$ gravitinos.
}

the inflaton energy scale, cannot be considered as a threat for nucleosynthesis. Their overproduction can be easily understood from the presence of a second derivative of the superpotential with respect to the super fields in the equation of motion for the helicity $\pm 1 / 2$ gravitinos. This gives rise to an effective mass for the helicity $\pm 1 / 2$ gravitinos, which is equivalent to the mass of the fermionic component of the inflaton, known as inflatino. The statement is true only if the inflaton sector has a single multiplet. In some sense helicity $\pm 1 / 2$ states eat the mass of the goldstino, which is related to the inflatino by an appropriate phase. These states remember their goldstino nature and this is the reason why they are produced very efficiently compared to the helicity $\pm 3 / 2$ states. In this paper we have argued that the same goldstino nature come into rescue the late decay of the helicity $\pm 1 / 2$ gravitino. It has been argued by many authors that the helicity $\pm 1 / 2$ gravitinos effectively behave like goldstino just after couple of inflaton oscillations. This, together with a requirement that the inflaton must decay to give a successful nucleosynthesis, leads to an efficient decay of the goldstino, or, the helicity $\pm 1 / 2$ gravitinos. Thus, they must not survive until nucleosynthesis, and hence they should not be considered as a threat to nucleosynthesis. This argument holds perfectly well for a single chiral field where the goldstino is inflatino with some additional phase. However, the extension of this argument in some models where there are more than one sectors of supersymetry breaking can be made applicable, provided supersymmetry breaking scale is still dominated by the inflaton energy density. Such a situation can arise if there exists a Polonyi field in the hidden sector, which we have briefly discussed. However, we still lack a complete formal tools to explore all possibilities such as mixing between the fermionic components of the inflaton sector and the Polonyi sector. This can in principle change the abundance of the helicity $\pm 1 / 2$ component of the gravitinos and a detailed study is certainly required in this direction.

It is important to note that the above discussion does not apply to the helicity $\pm 3 / 2$ gravitinos. The production of these states during preheating is always Planck mass suppressed and their existence is also independent of the goldstino, so they decay quite late. Due to time varying nature of their masses it is always hard to estimate their decay rate. It is also true that the helicity $\pm 3 / 2$ states are in general produced in less abundance than helicity $\pm 1 / 2$ states, however, their abundance cannot be neglected as pointed out in Refs. [6,11]. For a single multiplet they are the only genuine threat to Big Bang nucleosynthesis.

\section{ACKNOWLEDGEMENTS}

The authors are thankful to Antonio Maroto and Lev Kofman for useful discussions. The work of R.A was supported by "Sonderforchschungsbereich 375 für Astro- 
Teilchenphysik" der Deutschen Forschungsgemeinschaft.

[1] A. H. Guth, Phys. Rev. D 23, 347 (1981).

[2] E. F. Bunn, D. Scott and M. White, Ap. J. L 9, 441 (1995).

[3] A. Albrecht, P. J. Steinhardt, M. S. Turner and F. Wilczek, Phys. Rev. Lett. 48, 1437 (1982); A. D. Dolgov and A. D. Linde, Phys. Lett. B 116, 329 (1982); L. F. Abbott, E. Fahri and M. Wise, Phys. Lett. B 117, 29 (1982).

[4] J. Traschen and R. Brandenberger, Phys. Rev. D 42, 2491 (1990); L. A. Kofman, A. D. Linde and A. A. Starobinsky, Phys. Rev. Lett. 73, 3195 (1994); Phys. Rev. D 56, 3258 (1997); Y. Shtanov, J. Traschen and R. Brandenberger, Phys. Rev. D 51, 5438 (1995).

[5] A. D. Dolgov and D. P. Kirilova, Sov. J. Nucl. Phys. 51, 172 (1990); J. Baacke, K. Heitmann and C. Patzold, Phys. Rev. D 58, 7815 (1998); P. Greene and L. Kofman, Phys. Lett. B 448, 6 (1999).

[6] A. L. Maroto and A. Mazumdar, Phys. Rev. Lett 84, 1655 (2000).

[7] D. Bailin and A. Love, Supersymmetric Gauge Field Theory and String Theory, (IOP, Bristol 1994).

[8] R. Kallosh, L. Kofman, A. Linde and A. Von Proeyen, Phys. Rev D 61, 103503 (2000); G. F. Giudice, I. Tkachev and A. Riotto, JHEP 9908, 009 (1999).

[9] G.F. Giudice, A. Riotto and I. Tkachev, JHEP 9911, 036 (1999).

[10] A. L. Maroto and J. R. Pelaez, Phys. Rev. D 62, 023518 (2000).

[11] M. Bastero-Gil and A. Mazumdar, Phys. Rev. D 62, 083510 (2000).

[12] R. Kallosh, L. Kofman, A. Linde and A. Von Proeyen, hep-th/0006179.

[13] J. Cline and S. Raby, Phys. Rev. D 43, 1781 (1991).

[14] See, for example S. Sarkar, Rept. Prog. Phys. 59, 1493 (1996).

[15] S. Weinberg, Phys. Rev. Lett. 48, 1303 (1982).

[16] H. Pagel and J. R. Primack, Phys. Rev. Lett. 48, 223 (1982).

[17] J. Ellis, J. E. Kim and D. V. Nanopolous, Phys. Lett. B 145, 181 (1984); J. Ellis, D. V. Nanopolous, S. Sarkar, Nucl. Phys. B 259, 175 (1986).

[18] G. G. Ross and S. Sarkar, Nucl. Phys. B 461, 597 (1996).

[19] P. Fayet, Phys. Lett. B 175, 471 (1986).

[20] T. Gherghetta, Nucl. Phys. B 485, 25 (1997).

[21] M. Kawasaki and T. Moroi, Prog. Theor. Phys. 93, 879 (1995).

[22] D. H. Lyth, Phys. Lett. B 469, 69 (1999); 488, 417

(2000); D. H. Lyth and H. B. Kim, hep-ph/0011262. 